\title{
La greffe de conjonctif enfouie : comment améliorer la prévisibilité du recouvrement?
}

\section{Subepithelial connective tissue graft : how to improve the predictability of root coverage?}

MOTS-CLEFS :
- Recouvrement
radiculaire,
greffe de tissu conjonctif
enfouie,
récession gingivale.

\section{KEYWORDS:}

- Root coverage, subepithelial connective tissue graft,

gingival recession.

AOS 2014;267:35-39

DOI: $10.1051 / \mathrm{aos} / 2014206$

C) EDP Sciences 2014

\section{Résumé}

Dans le domaine du recouvrement radiculaire, la technique de la greffe de conjonctif enfouie est considérée, à juste titre, comme le gold stantard car elle permet un épaississement gingival et offre des résultats jugés généralement plus esthétiques, plus prévisibles et plus stables à long terme. Cependant, la prévisibilité des résultats obtenus par cette technique dépend de plusieurs facteurs regroupés et discutés dans cet article.

\begin{abstract}
In the area of root coverage, the technique of subgingival connective tissue graft is considered as the "Gold Stantard» because it allows gingival thickening and provides results considered generally more aesthetic, more predictable and stable on the long term. However, the predictability of the results obtained by this technique depends on several factors which are grouped and discussed in this work.
\end{abstract}

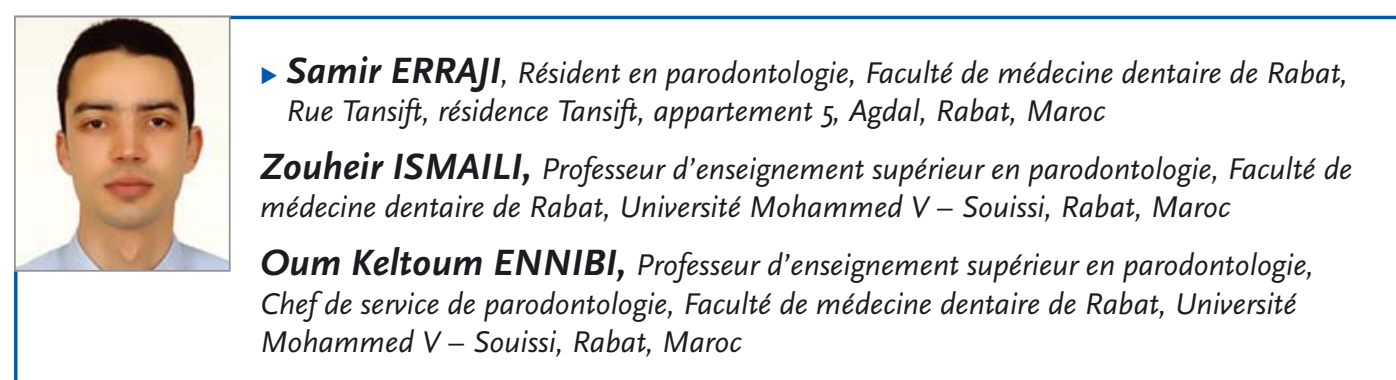




\section{INTRODUCTION}

Le recouvrement des récessions tissulaires marginales reste un challenge thérapeutique majeur face aux demandes esthétiques et fonctionnelles des patients. Ces dernières décennies, plusieurs techniques chirurgicales ont été évaluées dans de nombreuses études afin de tester leur prévisibilité de recouvrement radiculaire (RR).

Il est important de faire la distinction entre le succès et la prévisibilité d'une technique de RR. Le succès se définit par le pourcentage de RR moyen atteint, alors que la prévisibilité se définit par le pourcentage de $R R$ complet atteint [1].

La technique de RR par greffe de tissu conjonctif enfouie présente une excellente prévisibilité [2-5]. Néanmoins, plusieurs facteurs liés au patient, au défaut gingival et à son environnement mucogingival, ainsi qu'à la procédure chirurgicale peuvent influencer les résultats cliniques et doivent par conséquent être pris en considération.

\section{Facteurs liés au patient}

Ils sont représentés par le contrôle de plaque, le tabac et l'état général. En effet, un contrôle de plaque individuel insuffisant ou iatrogène - associant un brossage vigoureux, une brosse trop dure et une méthode horizontale - influence négativement les résultats du RR [6]. Aucune thérapeutique visant à corriger les récessions gingivales ne peut être envisagée avant le contrôle de l'inflammation gingivale et l'information du patient sur des techniques d'hygiène efficaces et non traumatogènes [7].

Plusieurs études ont rapporté l'effet négatif du tabac sur les résultats de RR obtenus par des procédures utilisant le conjonctif enfoui et leur stabilité dans le temps [8-10]. Kenneth et al. (2006) ont montré une différence statiquement significative concernant le taux de RR complet à six mois $(98,3 \%$ chez les patients non fumeurs contre $82,3 \%$ chez les patients fumeurs [entre 10 et 20 cigarettes/j]). Ils ont recommandé par conséquent l'arrêt ou la diminution du tabagisme pour une meilleure prévisibilité du traitement [11].

Sur le plan général, un diabète non équilibré interfère avec la cicatrisation parodontale et influence les résultats du RR notamment avec le conjonctif enfoui [12].

\section{Facteurs liés au défaut gingival et à l'environnement mucogingival}

Le niveau de support parodontal interdentaire est un facteur déterminant dans la prévisibilité des résultats de RR. Les récessions de classes I et II de Miller (1985) ayant un support parodontal interdentaire intact peuvent être totalement recouvertes avec une grande prévisibilité [13]. Toutefois, une étude récente de Aroca et al. (2010) rapporte un RR complet de récessions de classe III de Miller dans 38 \% des cas traités avec la technique du tunnel modifié décrite par Azzi et Étienne (1998) [14] avec ou sans adjonction des dérivés de la matrice amélaire [15].

La dimension de la récession semble être un facteur important à évaluer. En effet, le recouvrement des lésions larges $(>3 \mathrm{~mm}$ ) et profondes $(>5 \mathrm{~mm})$ est plus difficile à obtenir en raison de la plus grande surface dénudée avasculaire qui en résulte [6].

L'influence de la largeur et de la hauteur corono-apicale de la papille interdentaire a été également étudiée. Cependant, elle reste non consensuelle. En effet, Saletta et al. (2001) ont montré que plus la papille est courte, plus la probabilité d'obtenir un RR complet est grande [16]. Ce résultat peut être expliqué par le fait que les papilles courtes sont généralement associées à un biotype parodontal épais qui améliore le pronostic de recouvrement [17], alors que Haghighati et al. (2009) ont rapporté que plus la papille est haute et large, plus le RR complet est important. En outre, une papille de hauteur de $5 \mathrm{~mm}$ était constamment associée à un RR complet [18].

La position de la dent sur l'arcade est aussi un facteur à prendre en compte lors du RR. En effet, une vestibulo-version ou une proéminence exagérée d'une dent rend les résultats moins prévisibles [7] (fig. 1).

La quantité et l'épaisseur du tissu kératinisé peuvent aussi influencer le résultat du RR : un tissu kératinisé résiduel épais en grande quantité est perçu comme favorable [19]. Certains cliniciens se basent sur ce facteur pour décider de rajouter un greffon conjonctif ou non aux lambeaux déplacés. Cependant, l'évidence scientifique supportant cette approche reste limitée [20].

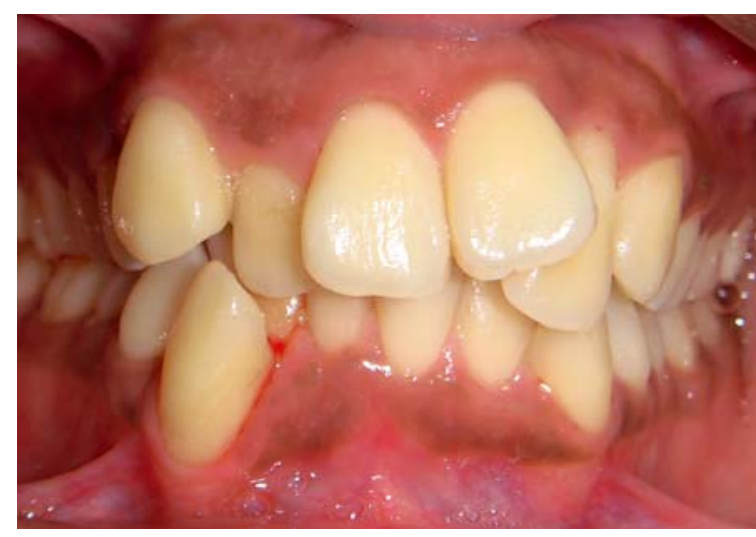

$\triangle$ Figure 1 :

Récession au niveau de la 43 en position très vestibulaire. 
Enfin, le recouvrement des racines présentant une lésion non carieuse ou précédemment cariées ou obturées par verres ionomères $[21,22]$ semble donner des résultats similaires à ceux obtenus avec des racines intactes.

\section{Facteurs liés à la procédure chirurgicale}

Différentes techniques associées à la GCE ont été proposées. En effet, le greffon peut être enfoui sous un lambeau d'épaisseur partielle déplacé plus ou moins coronairement [23], il peut également être enfoui, soit sous un lambeau déplacé latéralement [24], soit dans une enveloppe préparée autour de la récession [25]. Selon les revues systématiques et les méta-analyses, la technique associant le lambeau déplacé coronairement et la GCE est la technique qui donne le plus de RR complet et donc la plus prévisible dans le traitement des classes I et II de Miller [2-5, 20, 26].

L'efficacité clinique du conditionnement chimique de la racine par l'acide citrique ou le chlorhydrate de tétracycline n'a jamais pu être prouvée [7]. Récemment, l'utilisation du laser pour préparer la racine n'a pas pu améliorer les résultats du RR associé à la greffe de conjonctif enfouie [27]. Un surfaçage doux semble être suffisant pour préparer l'aire de recouvrement [7]. Une étude contrôlée et randomisée de Pini Prato et al. (1999) suggèrent que le surfaçage peut être facultatif. Il pourrait être remplacé par un simple polissage dans le cas de récessions étroites liées à un brossage traumatogène chez une population à haut niveau d'hygiène orale [28].

L'épaisseur du lambeau intervient dans la qualité du recouvrement. Une étude clinique de Baldi et al. (1999) a montré qu'une épaisseur du lambeau supérieure à $0,8 \mathrm{~mm}$ était associée à un RR complet. De plus, l'analyse par régression linéaire a révélé que chaque augmentation de 0,1 $\mathrm{mm}$ dans l'épaisseur du lambeau réduit la récession d'environ $0,2 \mathrm{~mm}$ dans tous les sites traités [29]. Récemment, une revue systématique réalisée par Hwang \& Wang (2006) a montré qu'un minimum de $1,1 \mathrm{~mm}$ est nécessaire pour obtenir un RR complet [30].

Cette épaisseur minimale fait que la réalisation d'un lambeau d'épaisseur totale peut être recommandée en cas de gencive très fine. Une étude de Mazzocco et al. (2011) a montré que l'épaisseur totale ou partielle du lambeau n'affecte pas la quantité de recouvrement [31].

Le greffon conjonctif lui aussi doit obéir à certaines mesures. Une épaisseur du greffon de 1,5 $\mathrm{mm}$ était jugée idéale [6]. Cependant, si le résultat de RR est satisfaisant, le résultat esthétique, lui, est médiocre à cause de l'épaisseur trop importante. Récemment, des modifications de la technique concernant l'épaisseur, la taille et le positionnement du greffon ont été proposées. Selon Zucchelli (2003), la largeur du greffon conjonctif doit être supérieure de $6 \mathrm{~mm}$ par rapport à la largeur de la récession mesurée à la jonction amélo-cémentaire (JAC). Sa dimension apico-coronaire est égale à la distance entre la JAC et la crête osseuse diminuée de la hauteur pré-opératoire du tissu kératinisé, une épaisseur de $1 \mathrm{~mm}$ est conseillée. Le greffon est positionné apicalement de la JAC, d'une distance égale à la hauteur pré-opératoire du tissu kératinisé [32]. Le RR avec ces nouvelles modifications reste le même, mais avec un rendu esthétique meilleur [32] (fig. 2a ; 2b). Le prélèvement peut être effectué selon différentes techniques conservant ou non une bande d'épithélium. Selon Byun et al. (2009), la préservation d'une collerette épithéliale lors du prélèvement n'intervient pas dans le résultat du RR [33].
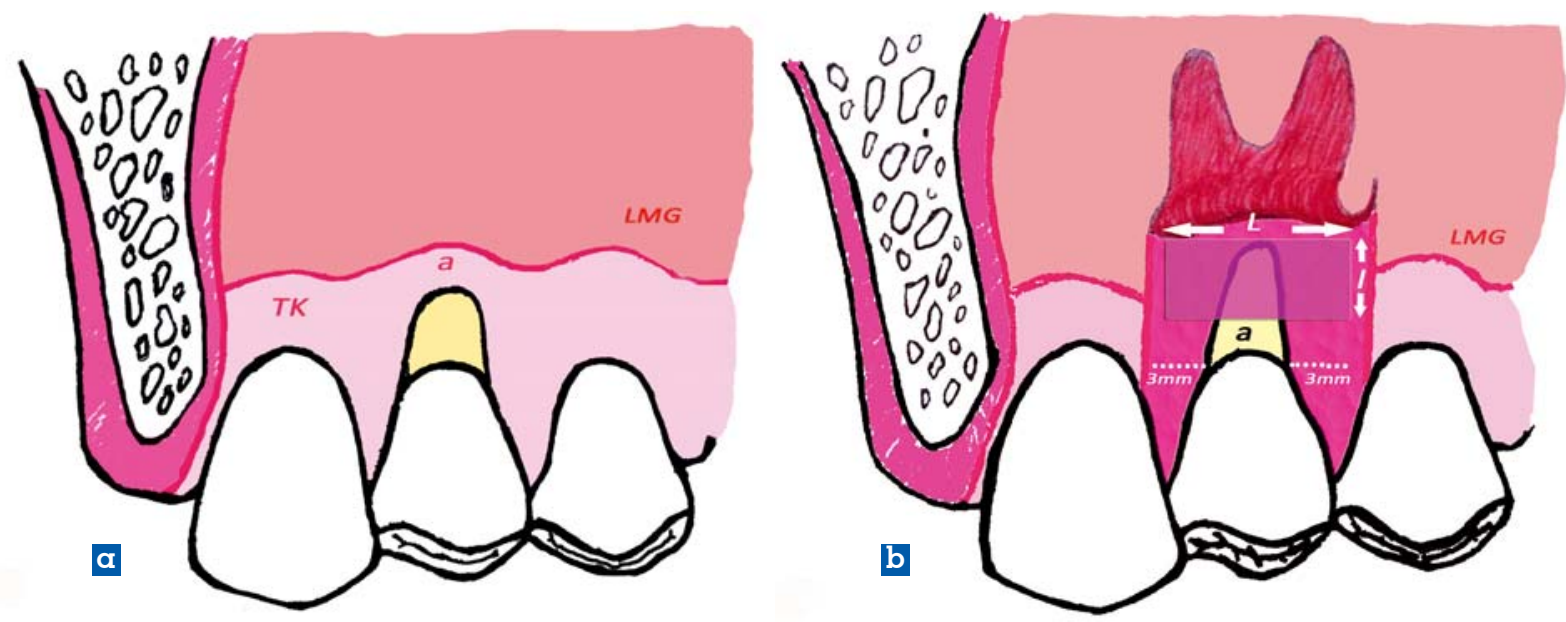

$\triangle$ Figure 2 :

(a) Situation clinique préopératoire. (b) Positionnement du greffon conjonctif selon Zucchelli.

TK : Tissu Kératinisé, LMG : Ligne Muco-Gingivale, a : Tissu kératinisé apical à la récession.

$\mathrm{L}:$ Largeur du greffon $=$ Largeur de la récession à la $\mathrm{JAC}+6 \mathrm{~mm}$,

1 : Dimension apico-coronaire du greffon = la profondeur de la récession - la hauteur préopératoire du TK. 
L'orientation du greffon selon sa face subépithéliale ou périostée ne semble pas avoir d'effet significatif sur le résultat clinique du recouvrement radiculaire ou de l'augmentation de hauteur gingivale [34]. Cependant, elle pourrait influencer la nature de l'attache après cicatrisation. En effet, selon Goldstein et al. (2001), le côté périosté peut agir comme une barrière qui empêcherait le contact direct du tissu conjonctif avec la surface radiculaire. Cela permettrait aux cellules du ligament parodontal de recoloniser la racine avant les cellules du tissu greffé et améliorerait par conséquent la formation d'une nouvelle attache conjonctive [35] (fig. 3).

La tension au niveau du lambeau a été étudiée par Pini Prato et al. (2000) qui ont trouvé que les sites avec une tension résiduelle entre 4 et $7 \mathrm{~g}$ au niveau du lambeau ont un taux de RR plus faible par rapport aux sites sans tension résiduelle [36] (fig. 4).

La position du lambeau par rapport à la JAC intervient dans le taux de RR complet. Selon Pini Prato et al. (2005), le rebord marginal du lambeau doit se situer au moins à $2 \mathrm{~mm}$ coronairement de la JAC [37] (fig. 5a ; 5b).

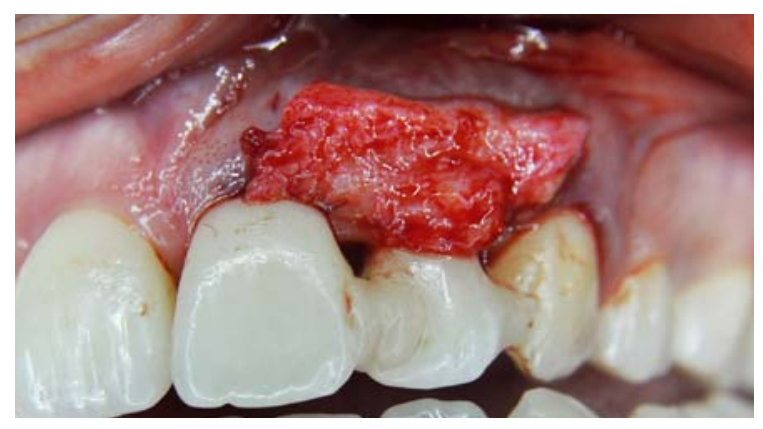

$\triangle$ Figure 3 :

Essayage du greffon, sa face périostée orientée vers la racine

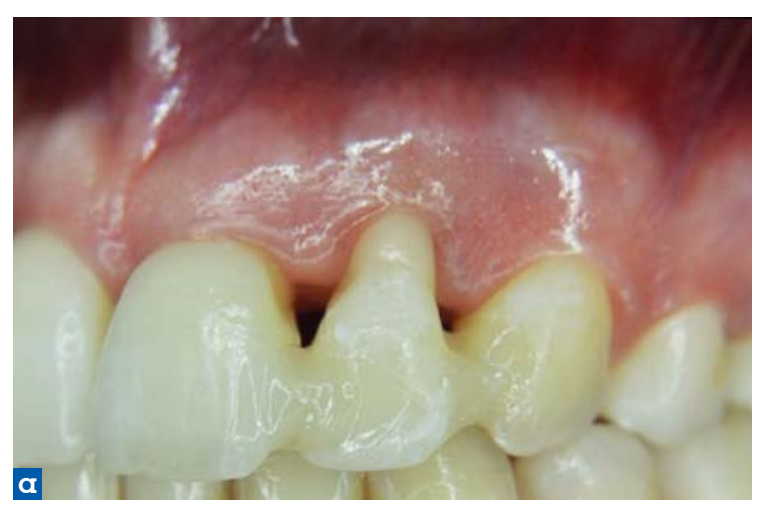

$\triangle$ Figure 5 :

Situation du lambeau par rapport à la JAC. (a, b): pré et postopératoire.
L'habileté du chirurgien doit aussi être prise en compte dans les résultats [8]. En effet, l'expérience de l'opérateur pourrait expliquer la variabilité des résultats thérapeutiques. Une étude clinique dont le but était d'évaluer l'efficacité de la technique de l'enveloppe entre trois opérateurs ayant une expérience professionnelle différente a montré cependant des résultats similaires [38].

L'approche microchirurgicale améliore sensiblement la vascularisation des greffes et le taux de RR par rapport à l'application d'une approche conventionnelle macroscopique [39].

\section{CONCLUSION}

La greffe du conjonctif enfouie est de plus en plus utilisée malgré la difficulté technique, du fait non seulement de son rendu esthétique excellent mais aussi de son taux de recouvrement important. Cependant, la prévisibilité des résultats dépend de la sélection du patient, du défaut gingival et d'une bonne maîtrise de la technique chirurgicale par le praticien.

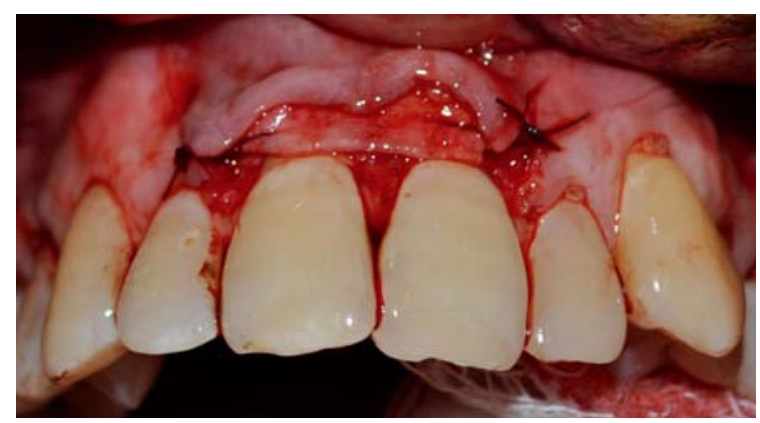

$\triangle$ Figure 4 :

Dissection d'un lambeau sans tension en vue de recouvrir totalement le greffon conjonctif et la récession.

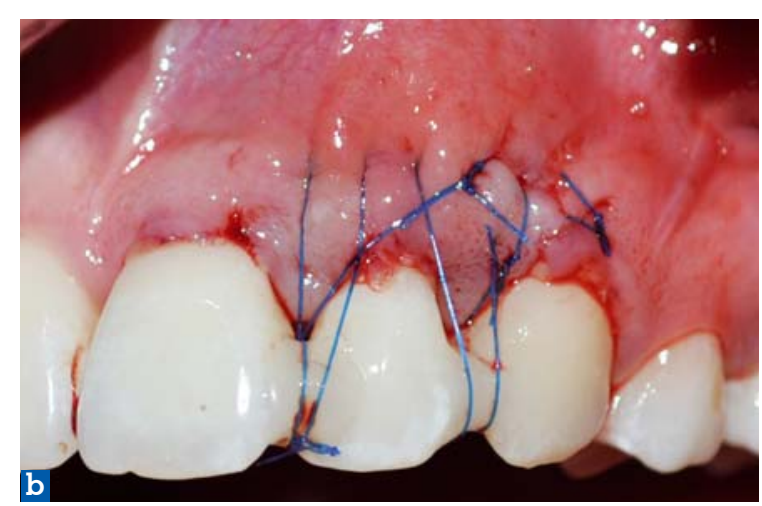


Bibliographie

[1] Paulo MC, Philip RM, Barrie KE. The use of free gingival grafts for aesthetic purposes. Periodontol 2000. 2001;27:72-96.

[2] Roccuzzo M, Bunino M, Needleman I, Sanz M. Periodontal plastic surgery for treatment of localized gingival recessions: a systematic review. J Clin Periodontol 2002;29:178-194.

[3] Oates TW, Robinson M, Gunsolley JC. Surgical therapies for the treatment of gingival recession. A systematic review. Ann Periodontol 2003;8:303-320.

[4] Cairo F, Pagliaro U, Nieri M. Treatment of gingival recession with coronally advanced flap procedures: a systematic review. J Clin Periodontol 2008;35:136-162.

[5] Chambrone L, Sukekava F, Arau'jo MG, Pustiglioni FE, Chambrone LA, Lima LA. Rootcoverage procedures for the treatment of localized recession-type defects: a cochrane systematic review. J Periodontol 2010;81:452-478.

[6] Wennström JL, Zucchelli G, Pini Prato GP. Clinical periodontology and implant dentistry. $5^{\mathrm{e}}$ ed.; chapitre 44 : Mucogingival Therapy Periodontal Plastic Surgery: p. 991. Oxford; Blackwell, 2008.

[7] Borghetti, V. Monnet-Corti. Chirurgie plastique parodontale. $2^{e} e ́ d$. "Conditions de succès dans le recouvrement radiculaire» $\mathrm{p}$ 264. Paris : CdP, 2008.

[8] Souza SL, et al. Subepithelial connective tissue graft for root coverage in smokers and nonsmokers: a clinical and histologic controlled study in humans. J Periodontol 2008;79(6):1014-1021.

[9] Andia DC, Martins AG, Casati MZ, Sallum EA, Nociti FH. Root coverage outcome may be affected by heavy smoking: a 2-year follow-up study. J Periodontol 2008;79(4):647-653.

[10] Martins AG, Andia DC, Sallum AW, Sallum EA, Casati MZ, Nociti Júnior FH. Smoking may affect root coverage outcome: a prospective clinical study in humans. J Periodontol 2004;75(4):586-591.

[11] Erley KJ, Swiec GD, Herold R, Bisch FC, Peacock ME. Gingival Recession Treatment With Connective Tissue Grafts in Smokers and NonSmokers. J Periodontol 2006;77:1148-1155.

[12] Al-Zahrani MS, Bissada NF. Predictability of connective tissue grafts for root coverage: clinical perspectives and a review of the literature. Quintessence Int 2005;36:609-616.

[13] Miller PD. A classification of marginal tissue recession. Int J Periodontics Restorative Dent 1985;5: 9-13.

[14] Azzi R, Étienne D. Recouvrement radiculaire et reconstruction papillaire par greffon conjonctif enfoui sous un lambeau vestibulaire tunnélisé et tracté coronairement. Journal de Parodontologie et d'Implantologie Orale 1998;17: 71-77.
[15] Aroca S, et al. Treatment of class III multiple gingival recessions: a randomized-clinical trial. J Clin Periodontol 2010;37:88-97.

[16] Saletta D, Pini Prato GP, Pagliaro U, Baldi

C, Mauri M, Nieri M. Coronally advanced flap procedure: is the interdental papilla a prognostic factor for root coverage? J Periodontol 2001;72:760-766

[17] Olsson M, Lindhe J. Periodontal

characteristics in individuals with varying form of the upper central incisors. J Clin Periodontol 1991;18:78-82.

[18] Haghighati F, Mousavi M, Moslemi N, Kebria MM, Golestan B. Acomparative study of two rootcoverage techniques with regard to interdental papilla dimension as a prognostic factor. Int J Periodontics Restorative Dent 2009;29:179-189.

[19] Wennström JL, Zucchelli G. Increased gingival dimensions. A significant factor for successful outcome of root coverage procedures? A 2-year prospective clinical study. J Clin Periodontol 1996;23:770-777.

[20] Cortellini P, Pini Prato G. Coronally advanced flap and combination therapy for root coverage. Clinical strategies based on scientific evidence and clinical experience. Periodontol 2000. 2012;59:158-184.

[21] Santamaria MP, Ambrosano GM, Casati MZ, Nociti Júnior FH, Sallum AW, Sallum EA. Connective tissue graft plus resin-modified glass ionomer restoration for the treatment of gingival recession associated with non-carious cervical lesion: a randomized-controlled clinical trial. J Clin Periodontol 2009;36(9):791-798.

[22] Goldstein M, Nasatzky E, Goultschin J, Boyan $\mathrm{BD}$, Schwartz Z. Coverage of previously carious roots is as predictable a procedure as coverage of intact roots. J Periodontol 2002;73(12):1419-1426.

[23] Langer B, Langer L. Subepithelial connective tissue graft technique for root coverage. $J$ Periodontol 1985;56(12):715-720.

[24] Nelson SW. The subpedicle connective tissue graft. A bilaminar reconstructive procedure for the coverage of denuded root surfaces.

J Periodontol 1987; 58(2):95-102.

[25] Raetzke PB. Covering localized areas of root exposure employing the "envelope" technique. J Periodontol 1985;56(7):397-402.

[26] Chambrone L, Chambrone D, Pustiglioni FE, Chambrone LA, Lima LA. Can subepithelial connective tissue grafts be considered the gold standard procedure in the treatment of Miller Class I and II recession-type defects? J Dent 2008;36:659-671.
[27] Dilsiz A, Aydin T, Yavuz MS. Root surface biomodification with an Er:YAG laser for the treatment of gingival recession with subepithelial connective tissue grafts. Photomed Laser Surg 2010;28(4):511-517.

[28] Pini Prato GP, et al. Coronally advanced flap procedure for root coverage. Treatment of root surface: root planning versus polishing. J Periodontol 1999;70:1064-1076.

[29] Baldi C, et al. Coronally advanced flap procedure for root coverage. Is flap thickness a relevant predictor to achieve root coverage? A 19-case series. J Periodontol 1999;70:1077-1084.

[30] Hwang D, Wang HL. Flap thickness as a predictor of root coverage: a systematic review. J Periodontol 2006;77(10):1625-1634.

[31] Mazzocco F, Comuzzi L, Stefani R, Milan Y, Favero G, Stellini E. Coronally advanced flap combined with a subepithelial connective tissue graft using full- or partial-thickness flap reflection. J Periodontol 2011;82(11):1524-1529.

[32] Zucchelli G, Amore C, Sforza NM, Montebugnoli L, De Sanctis M. Bilaminar techniques for the treatment of frecession-type defects. A comparative clinical study. J Clin Periodontol 2003;30: 862-870.

[33] Byun HY, Oh TJ, Abuhussein HM, Yamashita J, Soehren SE, Wang HL. Significance of the epithelial collar on the subepithelial connective tissue graft 2009;80(6):924-932.

[34] Lafzi A, Mostofi Zadeh Farahani R, Abolfazli $\mathrm{N}$, Amid R, Safaiyan A. Effect of connective tissue graft orientation on the root coverage outcomes of coronally advanced flap. Clin Oral Investig 2007;11(4):401-408.

[35] Goldstein M, Boyan BD, Cochran DL, Schwartz Z. Human histology of new attachment after root coverage using subepithelial connective tissue graft. J Clin Periodontol 2001;28:657-662.

[36] Pini Prato G, et al. Coronally advanced flap procedure for root coverage. Flap with tension versus flap without tension: A randomized controlled clinical study. J Periodontol 2000;71:188-201.

[37] Pini Prato GP, et al. Coronally advanced flap: The post-surgical position of the gingival margin is an important factor for achieving complete root coverage. J Periodontol 2005;76:713-722.

[38] Georges P, Nisand D, Etienne D, Mora F. Technique de l'enveloppe supra-périostée : étude clinique comparative préliminaire de son efficacité. Parodont Dent Rest 2009;29:201-211.

[39] Burkhardt R, Lang NP. Coverage of localized gingival recessions: comparison of micro- and macrosurgical techniques. J Clin Periodontol 2005;32(3):287-293. 